\title{
Novel Compounds for Increasing Women's Breast Size
}

\author{
Pyo Song1, Seonlin Kim², Dong-Chan Kim²,* \\ ${ }^{1}$ Department of Living Science Laboratory, MY Co. Ltd. Gyeonggi-do, 12721, South Korea. \\ ${ }^{2}$ Department of Novel Drug Design Laboratory, Neuronex, Goryeong, 40152, Korea. \\ Orcid: 0000-0002-9361-8023, *Corresponding Author
}

\begin{abstract}
This study aimed to elucidate compounds from natural plant extracts with the potential to expand women's breast size and boost growth. These compounds should also possess the ability to activate female hormone receptor without any adverse effects on the human body. Since it is traditionally known to be effective in female hormone control and health care, Leonurus japonicus was chosen as a source of compounds with potential female breast enlargement and skin's elasticity-enhancing activity. It was also expected that Leonurine, the main ingredient of the Leonurus japonicus, possessed such bioactivity. The L. japonicus extract was obtained using a polar solvent after hydrothermal extraction, and nano-micelling composition (NXCL-4950) was utilized to ensure penetration of the osmotic extract into the skin and increase blood circulation. Based on the composition of the extract, this study suggests the possibility of developing a new remedy, to be used in the beauty industry, that can improve skin health and elasticity and blood circulation for women, to particularly enhance the shape and texture of breasts.
\end{abstract}

\section{INTRODUCTION}

Warm-toned and firm breasts are almost every woman's desire. Most women with sagging or small breasts have major insecurities about their body. Obsession over severely small breast size can also lead to mental conditions, such as depression $[1,2]$. The structure of the breasts include mammary glands consisting of 15 to 20 irregular shaped lodes, each consisting of an alveolar gland, alveolar duct, and a literal duct [3]. Each lobe is supported by fatty tissue and dense conjunctival tissue, which is tightly coupled to the fascia of the pectoral muscle.

Estrogen is a female hormone known to maintain the shape of the mammary tissue (breast). The two major actions of female hormones related to mammary gland development increase the size of the reward line and the reward tube. It is reported that this activity promotes enlargement of the local breast tissue [4].

However, the synthesis of female hormones for medical purposes is required. It is noted that the administration of female hormones has several side effects. These side effects include breast cancer, high calcium blood pressure, jaundice, muwol, nausea, vomiting, diarrhea, headaches, edema, myocardial infarction, and stroke. In particular, such administration should be banned during pregnancy as it is reported to cause abnormal or irreversible damage to the fetus. The present study aimed to address the issue of medical estrogen preparation in a manner that results in minimal side effects. The purpose of this study was to provide a composition that can expand and increase the elasticity of women's breasts using natural herb extracts, with the function of female hormone receptor activation. In addition, modern medicine provides remedies that improve blood flow and vascular dilation of the breast tissue to help women achieve good breast health.

\section{MATERIALS AND METHODS}

\section{Herb Extract Preparation}

Natural herb extracts were prepared using hot water extraction and ethanol extraction. Cimicifuga heracleifoli (Black cohosh), Leonurus japonicus, Dioscorea japonica (yam), Puerariae radix, Acorus gramineus, and Glycine max were weighed, and then cut in the shade. Each dried herb was hydrothermally extracted at $85-100{ }^{\circ} \mathrm{C}$ for 3 to 5 hours or more with 2 to 10 times the weight of purified water. Each of the hydrothermal extracts was combined with ethanol 3 to 5 times the weight of the hydrothermal extract and subjected to secondary extraction for 20 to 30 hours or more to obtain ethanol extracts. The filtrate was obtained under reduced pressure using filter paper to prepare an extract concentrate using a vacuum rotary evaporator.. In addition to the ethanol extraction method, polar solvent extraction methods other than ethanol can also be used.

\section{Nano-micelles composition for breast enlargement}

The herb extract prepared using the above mentioned method was mixed with a solution of nano-mycelling formulation (NMF) (, Neuronex Co., Ltd.) [5]. The mixture was first mixed using a vortex for 5 minutes, and then ultrasonically stimulated for 3 minutes at a frequency of $40,000 \mathrm{~Hz}$. The micellar particle stabilization process was repeated 12 times using a liposofast (Avestin, Canada) nano extruder for 2 minutes. An electrical stimulation was applied at $80 \sim 100 \mathrm{~V}, 60 \mathrm{~mA}$ for 15 minutes, heated for $25 \sim 35$ minutes at $900 \sim 1,100{ }^{\circ} \mathrm{C}$, and vacuum dried for 7 hours in a dryer connected to nano-formulation system for the micelling composition formation. The nano-micelling composition can increase the ligand binding affinity of the plant bioactive compound binding site of estrogen better than the conventional plant extract concentrate.

\section{Gel formula for breast enlargement and skin elasticity}

A gel for female breast enlargement and skin elasticity improvement formulation included multiple components. The potentially therapeutic plant extract of the Cimicifuga heracleifoli (Black cohosh), Leonurus japonicus, Dioscorea 
japonica, Puerariae Radix, Acorus gramineus, and Glycine max was the active ingredient in the composition of this gel. The addition of a natural peptide functioned to stimulate skin fibroblasts to produce collagen, elastin, and other proteins in the matrix of the dermis. These included peptides such as keratin peptide, collagen peptide, or gelatin. These compounds also serve as a moisturizing agent to boost skin firmness and make it appear fuller. Moisturizing and nourishing agents included at least one of sodium hyaluronate, squalene, potassium cetyl phosphate, or Red Myrrh alcohol. However, this is merely exemplary, and the scope of the present invention is not limited thereto. Deionized water was added to improve consistency of the gel. Xanthan Gum and agarose agar extract were used as a conditioning and stabilizing agent, respectively. The gel included a composition of licorice (licorice root), Curcuma longa, grape, grapefruit, or bamboo extract, and grape seed oil. It also included at least one extract of raspberry, cinnamon, Hodenia dulcis, japonica, or alder extract or a mixture thereof to improve blood circulation and vasodilation.

\section{In silico molecular docking study}

For in silico molecular docking investigation, we used Autodock Vina [6] and Discovery Studio 3.5 version [7] for 3D structure analysis and Discovery Studio 3.5 version for $2 \mathrm{D}$ structure analysis. The estrogen receptor utilized a 3D structure (PDB ID $=1 \mathrm{R} 5 \mathrm{~K})$ registered at www.rcsb.org. As the control ligand substance bound to the estrogen receptor, (2E) -3- $\{4-$ [(1E) -1,2-DIPHENYLBUT-1-ENYL] PHENYL\} acrylic acid was used. The significance level of the ligand binding affinity of leonurine on the target protein was set at $*, p \leq 0.05$ versus control. All data are represented as the means \pm SEM (Standard Error of the Means).

\section{Breast enlargement (size up) test}

After applying the gel (cream) containing the female breast enlargement and skin elasticity enhancing composition to the breast, it was analyzed for size change after 21 days. 11 women who participated in the experiment were aged $20 \sim 35$ years and analyzed sequentially for 1 day, 3 days, 7 days, 14 days, 21 days after application the gel (cream). The significance level of the breast enlargement size was set at $*, p \leq 0.05$ versus control. All data were represented as the means \pm SEM (Standard Error of the Means).

\section{RESULTS}

\section{Leonurine binds to estrogen receptor active site}

The estrogen receptor utilized the 3D structure (PDB ID = $1 \mathrm{R} 5 \mathrm{~K})$ registered in www.rcsb.org. In addition, the estrogen binding ligand substance bound to the estrogen receptor (2E) 3- \{4-[(1E) -1,2-DIPHENYLBUT-1-ENYL] PHENYL\} and acrylic acid was used. To identify the possibility that leonurine, the main component of Leonurus japonicus, could influence estrogen receptor protein activity, the interaction between the leonurine ligand and target protein estrogen receptor was analyzed through in silico molecular investigation. Figure 1 shows a three-dimensional protein analysis image in which (2E) -3- $\{4-[(1 \mathrm{E}) \quad-1,2-D I P H E N Y L B U T-1-E N Y L] \quad$ PHENYL $\}$ acrylic acid is bound to the estrogen receptor and Figure 2 shows a two-dimensional protein analysis image in which (2E) -3- $\quad\{4-[(1 \mathrm{E}) \quad-1,2-D I P H E N Y L B U T-1-E N Y L] \quad$ PHENYL $\}$ acrylic acid is bound to PDB ID $=1 \mathrm{R} 5 \mathrm{~K}$ estrogen receptor. Figure 3 is a three-dimensional protein analysis image in which Leonurine, an active ingredient of Leonurus japonicus extract, is bound to an estrogen receptor. The average molecular binding affinity (docking energy) scores of the acrylic acid and leonurine on the estrogen target protein were $-6.74 \mathrm{kcal} / \mathrm{mol}$ (acrylic acid, SEM \pm 0.46 ) and $-6.15 \mathrm{kcal} / \mathrm{mol}$ (leonurine, SEM \pm 0.21 ) (Table 1). The numbers of the binding modes of both ligands to the estrogen receptor active site were nine $(n=9)$, respectively. Based on the present molecular docking data, leonurine appeared as a similar binder to the estrogen target protein with the (2E) -3- \{4-[(1E) -1,2-DIPHENYLBUT-1ENYL] PHENYL\} acrylic acid (Fig. 1 and Fig. 2). (a)

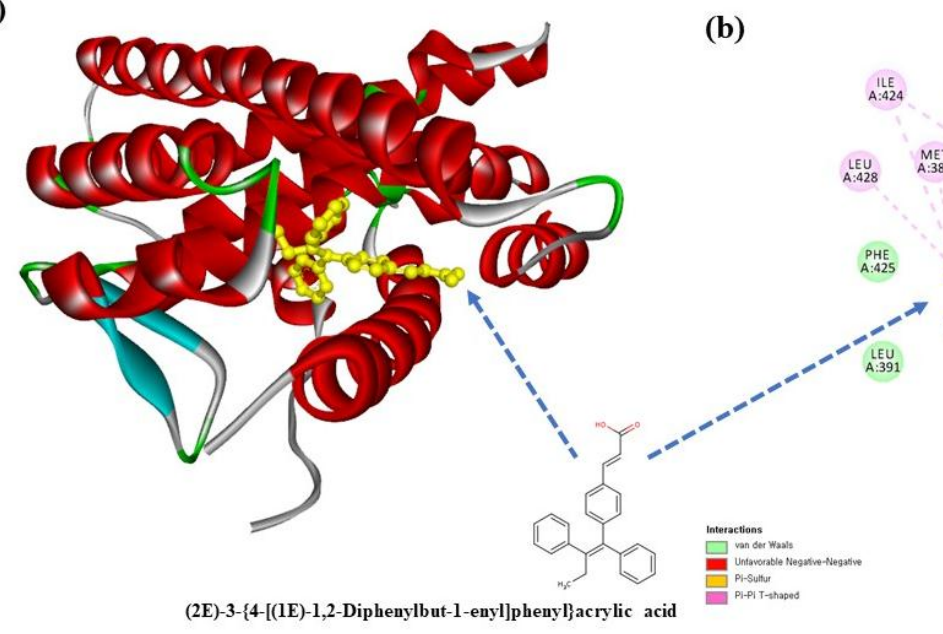

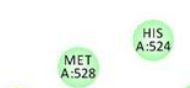

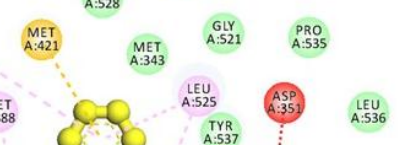




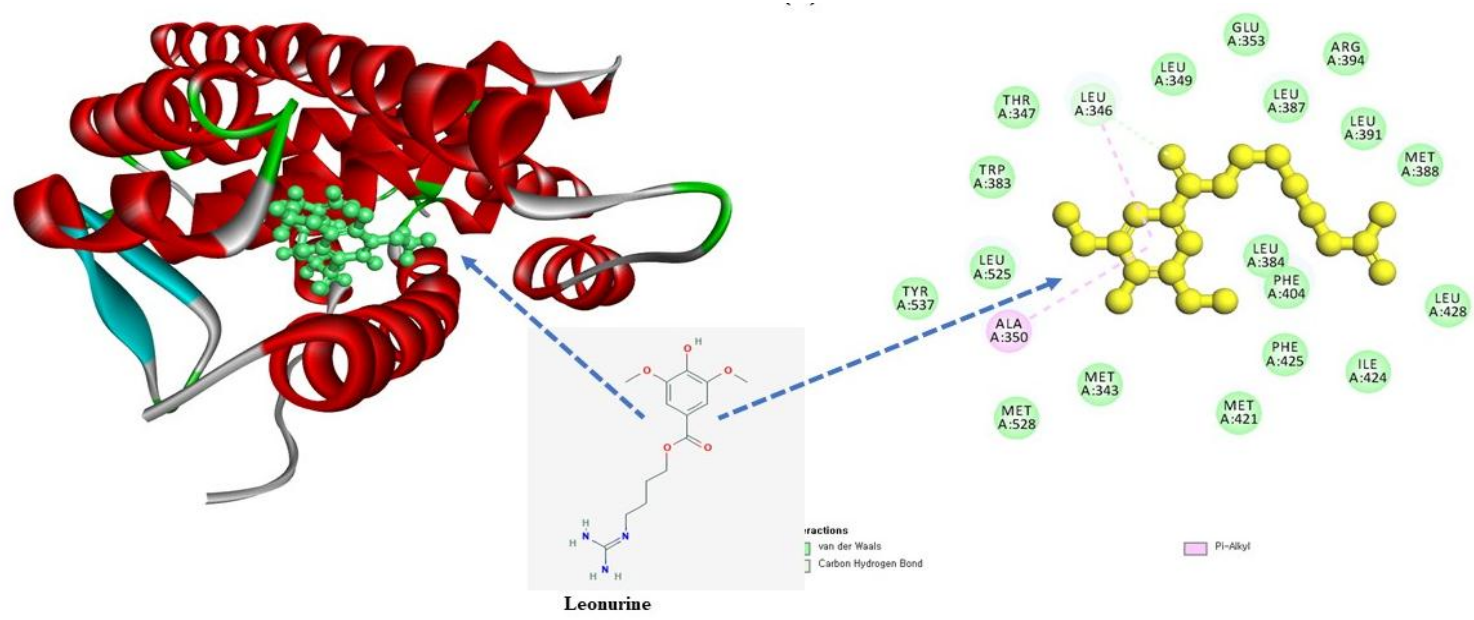

(a)

(b)

Figure 2: (a)Three-dimensional in silico molecular binding protein analysis images combined with leonurine at estrogen receptors. (b) Two-dimensional protein analysis images combined with leonurine at estrogen receptors.

Table 1: Binding affinity of the acrylic acid and leonurine on the estrogen receptor active site.

\begin{tabular}{|c|ccc|}
\hline & $\begin{array}{c}\text { Max Binding Affinity } \\
\text { (kcal/mol) }\end{array}$ & $\begin{array}{c}\text { Average Binding Affinity } \\
\text { (kcal/mol) }\end{array}$ & $\begin{array}{c}\text { SEM } \\
\text { (n=\# of binding } \\
\text { mode) }\end{array}$ \\
\hline $\begin{array}{c}\text { Acrylic acid on the } \\
\text { estrogen receptor }\end{array}$ & -7.5 & -6.74 & \pm 0.46 \\
$\begin{array}{l}\text { Leonurine on the } \\
\text { estrogen receptor }\end{array}$ & -6.4 & -6.15 & $\begin{array}{r}(\mathrm{n}=9) \\
\pm 0.21 \\
(\mathrm{n}=9)\end{array}$ \\
\hline
\end{tabular}

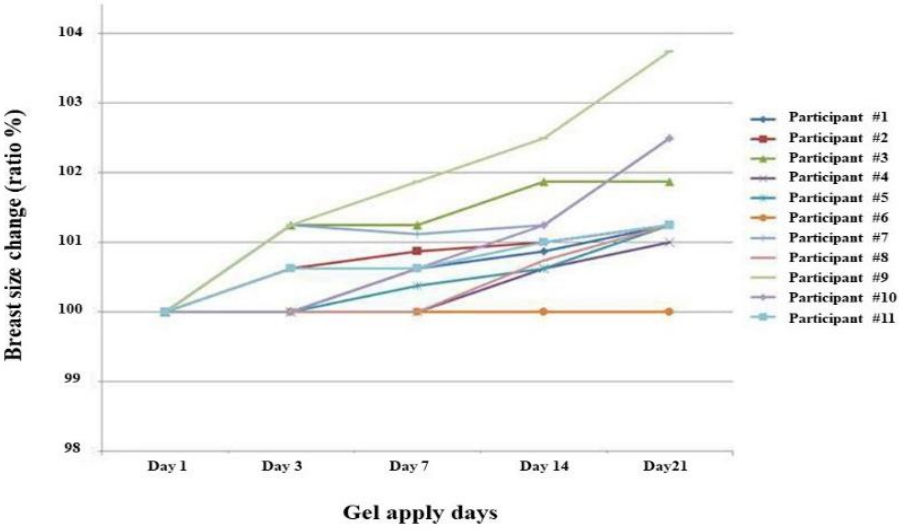

(a)

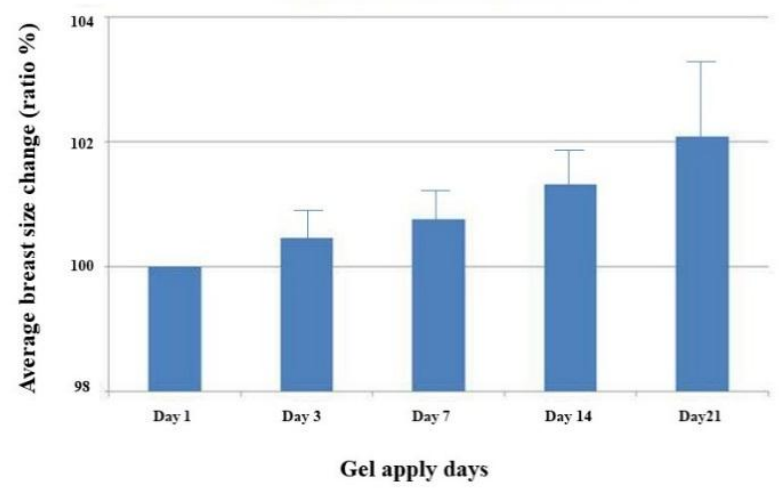

(b)

Figure 3: (a) The change in the breast size, 21 days after the application of the breast enlargement composition gel, of each participant. (b) The average value change pattern of the breast enlargement of the 11 applying volunteers. 


\section{Leonurine containing composition affects breast enlargement}

10 Participants show a change in breast size 21 days after applying a cream containing a female breast enlargement and skin elasticity enhancing composition according to an embodiment of the present invention to the breast (Fig. 3 and Fig. 4).

\section{DISCUSSION}

Normal breast enlargement occurs throughout the majority of a woman's life. It begins before you're born, ends at menopause, and has several stages in between.. Breast size varies significantly from one person to another. Throughout life, breasts undergo changes in development, which include monthly menstrual cycles and pregnancies. Each monthly cycle will cause changes in breasts due to hormonal stimulation. Breasts may become larger and painful during cycle.. During pregnancy, breasts begin preparing to produce milk for the baby, which is called lactation. This process will create several changes to breasts: areola swelling, darkening, and increasing in size, swollenness and soreness along the sides of breasts, and a tingling sensation in the nipples, blood vessels in breasts become more noticeable.

Estrogen levels change during the menstrual cycle, and this is enables the change of breast tissue. Most of birth control pills contain the estrogen, which is a synthetic form of progesterone[2]. Ingestion of this pill can stimulate the breasts to grow. There is another link between the birth control pill and breast enlargement in that changes to hormone levels can cause the body to retain fluid. When fluid retention occurs, breasts feel larger, and may return to normal when ingestion of the pill is stopped.

In this study, we found a functional composition that minimizes the negative effects on women's skin and physiological changes, and greatly enlarges the shape of women's breasts, and improves the elasticity of skin. According to the our experimental results, our novel herb mixture composition [8], made by effectively mixing natural medicinal ingredients and nano-micelling, was applied to 11 women's breasts for 21 days; it is possible that it may have affected the estrogen receptor activity and relatively enlarged the woman's breast size. Overall, leonurine, an active ingredient of Leonurus japonicus extract, which is likely to be the most active ingredient in the composition, was used in the present study and was found to bind to the estrogen receptor of breast cells and affect breast enlargement. If the composition developed in this study is utilized and further studied in more clinical trials[9], it is expected that more reliable and meaningful results will be obtained. Based on these results, it can be thought that it will be possible to develop a beauty product for breast enlargement that is safe for women's skin and can manage women's breast shape more safely and effectively.

\section{ACKNOWLEDGEMENT}

This research was supported by MY Co. Ltd. research fund (fund code: MY-20180601).

\section{REFERENCES}

[1] A.J.M. Cornelissen, S.M.H. Tuinder, E.M. Heuts, R. van der Hulst, J. Slatman, What does a breast feel like? A qualitative study among healthy women, BMC Womens Health, 18 (2018) 82.

[2] M.B.M. van Duursen, Modulation of estrogen synthesis and metabolism by phytoestrogens in vitro and the implications for women's health, Toxicol Res (Camb), 6 (2017) 772-794.

[3] D.F. Moffat, J.J. Going, Three dimensional anatomy of complete duct systems in human breast: pathological and developmental implications, J Clin Pathol, 49 (1996) 4852.

[4] S.C. Moore, C.E. Matthews, X. Ou Shu, K. Yu, M.H. Gail, X. Xu, B.T. Ji, W.H. Chow, Q. Cai, H. Li, G. Yang, D. Ruggieri, J. Boyd-Morin, N. Rothman, R.N. Hoover, Y.T. Gao, W. Zheng, R.G. Ziegler, Endogenous estrogens, estrogen metabolites, and breast cancer risk in postmenopausal Chinese women, J Natl Cancer Inst, 108 (2016)

[5] D.C. Kim, C.E. Lee, NXCL-4950, a novel composite applicable to peripheral skin, is capable of increasing skin temperature by enhancing capillary circulation, Clin Exp Dermatol, 38 (2013) 244-250.

[6] O. Trott, A.J. Olson, AutoDock Vina: improving the speed and accuracy of docking with a new scoring function, efficient optimization, and multithreading, J Comput Chem, 31 (2010) 455-461.

[7] S.N. Rath, D. Das, V.B. Konkimalla, S.K. Pradhan, In silico study of mirna based gene regulation, ilnvolved in solid cancer, by the assistance of argonaute protein, Genomics Inform, 14 (2016) 112-124.

[8] Y. Kakisaka, T. Ohara, H. Tozawa, S. Sato, S. Katayama, T. Suzuki, N. Hino-Fukuyo, S. Kure, Panax ginseng: a newly identified cause of gynecomastia, Tohoku J Exp Med, 228 (2012) 143-145.

[9] A. Adefisan, S. Owumi, O. Adaramoye, Root bark extract of Calliandra portoricensis (Jacq.) Benth. chemoprevents $\mathrm{N}$-methyl-N-nitrosourea-induced mammary gland toxicity in rats, J Ethnopharmacol, 233 (2019) 22-33. 